\title{
Impact of loneliness and depression on mortality: results from the Longitudinal Ageing Study Amsterdam
}

Tjalling J. Holwerda, Theo G. van Tilburg, Dorly J. H. Deeg, Natasja Schutter, Rien Van, Jack Dekker, Max L. Stek, Aartjan T. F. Beekman and Robert A. Schoevers

\section{Background}

Loneliness is highly prevalent among older people, has serious health consequences and is an important predictor of mortality. Loneliness and depression may unfavourably interact with each other over time but data on this topic are scarce.

\section{Aims}

To determine whether loneliness is associated with excess mortality after 19 years of follow-up and whether the joint effect with depression confers further excess mortality.

\section{Method}

Different aspects of loneliness were measured with the De Jong Gierveld scale and depression with the Centre for Epidemiologic Studies Depression Scale in a cohort of 2878 people aged 55-85 with 19 years of follow-up. Excess mortality hypotheses were tested with Kaplan-Meier and Cox proportional hazard analyses controlling for potential confounders.

\section{Results}

At follow-up loneliness and depression were associated with excess mortality in older men and women in bivariate analysis but not in multivariate analysis. In multivariate analysis, severe depression was associated with excess mortality in men who were lonely but not in women.

\section{Conclusions}

Loneliness and depression are important predictors of early death in older adults. Severe depression has a strong association with excess mortality in older men who were lonely, indicating a lethal combination in this group.

\section{Declaration of interest \\ None.}

\section{Copyright and usage}

(c) The Royal College of Psychiatrists 2016.
With globally increasing numbers of older people, social participation of older adults and the detrimental aspects of loneliness are receiving growing attention worldwide. Recent studies indicate that loneliness is associated with or predicts serious mental and physical health consequences such as suicidality, impaired cognition and dementia, coronary heart disease, diabetes, hypertension and metabolic syndrome. ${ }^{1-7}$ In an earlier community study among older adults we found that feelings of loneliness also predicted excess mortality, ${ }^{8}$ and a few other studies recently published similar results with loneliness predicting functional decline and death. ${ }^{9,10}$ The mechanisms by which loneliness affects mental and physical health remains largely unclear but health behaviours, excess stress reactivity, inadequate and inefficient physiological repair and maintenance processes are thought to play an important role in how loneliness has an impact on health. ${ }^{11}$ Similarly, depression is an established risk factor for excess mortality ${ }^{12}$ and the interplay of loneliness and depression should be examined in this context.

Loneliness has been classically defined as a subjectively experienced aversive emotional state that is related to the perception of unfulfilled intimate and social needs. ${ }^{13}$ Weiss suggested that there are two types of loneliness: social and emotional loneliness. ${ }^{14}$ Social loneliness occurs when the amount and quality of relationships with others is smaller than one desires. It concerns the subjective experience of the quality of relationships. Emotional loneliness occurs where the perceived intimacy in relationships is not satisfactory. Emotional and social loneliness are also referred to as intimate and relational loneliness, respectively. ${ }^{15}$ Emotional and social loneliness are distinct from social isolation, which can be defined in terms of a quantifiable reflection of the social network size and frequency and paucity of contacts. ${ }^{16}$ Loneliness has been ascertained using a single selfreport measure or by validated psychometric scales. ${ }^{1}$ Earlier studies often did not study differences between social loneliness and emotional loneliness. ${ }^{2-10}$ This distinction is important, as it may provide insight regarding the consequences of loneliness and may provide clues for possible interventions.

Previous research indicates that loneliness and depression are associated and often co-occur. ${ }^{1}$ Whether co-occurrence attributes to a higher mortality risk remains unclear. Until now, associations between loneliness and mortality have been studied in naturalistic cohort studies with follow-up periods ranging from 5 to 10 years for depression and up to 10 years for loneliness. ${ }^{8,17}$ Studies with a relatively long follow-up time are needed to study the longitudinal associations between exposure to loneliness, depression or the combined condition and eventual mortality rates. Also it may take time before the effects of loneliness and depression are revealed. However, research on long-term effects is scarce. ${ }^{18}$ Other studies have shown different health risk profiles for loneliness and depression in men and women. Some studies show that men who are lonely and depressed have higher excess mortality rates than women. ${ }^{8,12,17}$ Another study has shown loneliness to be associated with an increased risk of incident coronary heart disease in women and a review study showed depression to be more prevalent in women with coronary heart disease. ${ }^{3,19}$ Also, among women without a history of cardiovascular disease, depression was shown to be an independent predictor of early death. ${ }^{12}$ The mechanisms responsible for differences between men and women remain unclear but biological, psychosocial differences and differences in coping style between men and women possibly play a role. ${ }^{12}$ Therefore, considering gender differences is important in studying the outcomes of loneliness 
and depression. Earlier studies have also shown a dose-response relationship between depression severity and excess mortality. ${ }^{20}$ Therefore, it is important to also consider the depression severity level in mortality studies on loneliness and depression. The main objective of our study was to establish whether loneliness at baseline was associated with an increased chance of death after 19 years of follow-up and whether depression is further increasing this risk, possibly resulting in a toxic combination between loneliness and depression. We also assessed differences between different aspects of loneliness, between different levels of depression severity and between men and women.

\section{Method}

\section{Participants}

The Longitudinal Aging Study Amsterdam (LASA) is a prospective study on physical, emotional, cognitive and social functioning in the Dutch general population. The LASA cohort is based on a sample born between 1908 and 1937, initially aged 55-85, based in three representative geographic regions in The Netherlands. The sample was recruited from 11 municipal registries in 1992 for the Living Arrangements and Social Networks of Older Adults (LSN) programme with an oversampling of older people and men. The initial response rate was $62 \%(n=3805)$. On average 11 months after the LSN interview the 3677 surviving participants were approached for the first LASA cycle (1992-1993). Of the survivors 3107 participated yielding a response rate for the first LASA cycle of $85 \%$. Examinations consisted of an interview that was performed at home. Every 3 years the participants were re-examined. Details on data collection procedures have been described elsewhere. ${ }^{21}$ Of all participants of the interview 2878 were included in this study as 229 participants had no data on one or more of the baseline variables. Informed consent was obtained from all participants and the study was approved by the Medical Ethics Committee of the VU University Medical Center, Amsterdam. The Longitudinal Aging Study Amsterdam (LASA) was initiated by the Dutch Ministry of Welfare, Health and Culture (currently Ministry of Health, Welfare and Sports).

\section{Measures}

To examine the association between loneliness, depression and risk for all-cause mortality we used data from the municipal registry to determine the mortality status of participants. The reference date of mortality was 1 of September 2011. The survival time counted was 6916 days. Data are processed on an anonymous basis. Death certificates, supplied by the doctor, are sent to the medical official of Statistics Netherlands, which processes the data.

To assess loneliness we used the De Jong Gierveld loneliness scale, a validated 11-item scale which can be applied as a unidimensional scale. ${ }^{22}$ The scale items were developed using Weiss' distinction between social and emotional loneliness with emotional loneliness stemming from the absence of an intimate relationship or a close emotional attachment and social loneliness stemming from the absence of a broader group of contacts or an engaging social network. ${ }^{14}$ The number of item responses indicative for loneliness is counted (range $0-11$ ). The cut-off score for the loneliness scale is $\geqslant 3$. Individuals with scores $\geqslant 2$ on six emotional loneliness items are considered emotionally lonely and those with scores $\geqslant 2$ on five social loneliness items are considered socially lonely. ${ }^{23}$ The correlation between emotional and social loneliness is 0.29 (Spearman's rho) indicating that it is useful to make the distinction between emotional and social loneliness when measuring loneliness.
Depression was measured using the Centre for Epidemiologic Studies Depression Scale (CES-D), a 20-item scale developed to measure depressive symptoms in the community. ${ }^{24}$ The CES-D was originally designed for epidemiological survey in the general population. Studies have demonstrated that it has good psychometric properties in different populations and have shown its usefulness in physically ill and older populations. The sensitivity for major depressive disorder is $100 \%$ and the specificity $88 \%{ }^{25}$ The CES-D (range 0-60) has a standard cut-off of $\geqslant 16$ for depressive symptoms and $\geqslant 24$ for a severe level of depressive symptoms or diagnosis of a major depressive episode. ${ }^{26,27}$

The personal network was defined as the people with whom participants are in touch regularly and who are important to them. For the personal network, relationships were identified by name with a domain-specific approach with the following domains: household members, children and their partners, other relatives, friends, neighbours, colleagues from work (including voluntary work) or school, members of organisations. The number of people the participant had frequent contact with and who were important to the participant was counted. The definitions of frequent and important were left to the respondent. For all the respondents the partner, if there was one, was included in the network. Only individuals above the age of 18 were included. The age of 18 was chosen to exclude young children who are often not part of the potential support for a participant. The number of people (with a minimum of 0 and a maximum of 80) was taken as the size of the total network. ${ }^{28}$ If for personal network no data were available in the first LASA cycle, data from the LSN study were imputed.

Demographic variables and chronic diseases were assessed and are detailed in Table 1 (see online Table DS1 for a version that includes a larger number of variables). Chronic diseases were assessed by self-report, which has been shown as fairly accurate. ${ }^{29}$

General cognitive functioning was measured by the MiniMental State Examination (MMSE) ${ }^{30}$ Functional limitations were assessed by three questions about physical functioning (activities) derived from the Organisation for Economic Co-operation and Development (OECD) questionnaire, a disability assessment instrument in the general population. ${ }^{31}$ Participants could specify that they have no difficulties with physical functioning, could do the activities with difficulty, with help or were not able to perform these activities at all.

\section{Analyses}

In describing the study population descriptive statistics were used; $\chi^{2}$ statistics were used to describe differences between men and women. We calculated mortality hazard ratios (HRs) with 95\% confidence intervals using Cox proportional hazard regression models for the different aspects of loneliness, different depression severity levels and all other potential risk factors associated with excess mortality. Kaplan-Meier analyses were performed for the different aspects of loneliness and different depression severity levels. In multivariate analysis we first performed Cox proportional hazard regression analyses for the different aspects of loneliness and time to death adjusting for depression and the selected covariates (demographical factors (age, gender, marital status and educational level), network status, chronic diseases, global cognitive functioning and functional limitations). Second, we performed Cox proportional hazard regression analyses for the different depression severity levels adjusting for loneliness and the previously mentioned selected covariates. We performed subgroup analysis if an interaction was found between gender and the different aspects of loneliness, between gender and the different depression severity levels and between the 


\begin{tabular}{|c|c|c|c|c|}
\hline Characteristic & Total & Men & Women & $P^{a}$ \\
\hline Gender, $n$ (\%) & $2878(100)$ & $1397(48.5)$ & $1481(51.5)$ & \\
\hline Age, years: mean & 70.35 & 70.49 & 70.23 & \\
\hline Number of people in personal network, mean & 13.82 & 13.73 & 13.92 & \\
\hline Living together with partner, $n$ (\%) & $1877(65.2)$ & $1103(79.0)$ & $774(52.3)$ & 0.001 \\
\hline Living together with other, $n$ (\%) & $429(14.9)$ & $241(17.3)$ & $208(14.0)$ & 0.19 \\
\hline Lonely, $n$ (\%) & $873(30.3)$ & $397(28.4)$ & $476(32.1)$ & 0.03 \\
\hline Emotional loneliness, $n$ (\%) & $799(27.8)$ & $317(22.7)$ & $482(32.5)$ & 0.001 \\
\hline Social loneliness, $n$ (\%) & $664(23.1)$ & $353(25.3)$ & $311(21.0)$ & 0.007 \\
\hline Depressive disorder, $n$ (\%) & $390(13.6)$ & $139(9.9)$ & $251(16.9)$ & 0.001 \\
\hline Severe depressive disorder, $n$ (\%) & $129(4.5)$ & $41(2.9)$ & $88(5.9)$ & 0.001 \\
\hline Pulmonary disease, $n$ (\%) & $329(11.4)$ & $182(13.0)$ & $147(9.9)$ & 0.009 \\
\hline Cardiovascular disease, $n$ (\%) & $568(19.7)$ & $345(24.7)$ & $223(15.1)$ & 0.001 \\
\hline Peripheral artery disease, $n$ (\%) & $276(9.6)$ & $138(9.9)$ & $138(9.3)$ & 0.61 \\
\hline Diabetes, $n$ (\%) & $219(7.6)$ & $102(7.3)$ & $117(7.9)$ & 0.55 \\
\hline Cerebrovascular disease, $n$ (\%) & $145(5.0)$ & $90(6.4)$ & $55(3.7)$ & 0.001 \\
\hline Osteoarthritis, $n$ (\%) & $938(32.6)$ & $319(22.8)$ & $619(41.8)$ & 0.001 \\
\hline Rheumatoid arthritis, $n$ (\%) & $194(6.7)$ & $61(4.4)$ & $133(9.0)$ & 0.001 \\
\hline Cancer, $n(\%)$ & $261(9.1)$ & $92(6.6)$ & $169(11.4)$ & 0.001 \\
\hline Other diseases, $n(\%)^{b}$ & 947 (32.9) & $395(28.3)$ & 552 (37.3) & 0.001 \\
\hline Number of chronic diseases, ${ }^{c}$ mean & 1.38 & 1.27 & 1.49 & \\
\hline Mini-Mental State Examination score, mean & 27.11 & 27.15 & 27.1 & \\
\hline \multicolumn{5}{|l|}{ Functional limitations, $n$ (\%) } \\
\hline No difficulties with tasks & $1734(60.3)$ & $936(67.0)$ & $898(60.6)$ & 0.001 \\
\hline Tasks with difficulty & $547(19.0)$ & $253(18.1)$ & $294(19.9)$ & \\
\hline Tasks with help & $324(11.3)$ & $128(9.2)$ & $196(13.2)$ & \\
\hline Tasks not possible & $273(9.5)$ & $80(5.7)$ & $193(13.0)$ & \\
\hline
\end{tabular}

different aspects of loneliness and the different depression severity levels. Our specified threshold of significance was $P<0.05$. For interactions the threshold of significance was $P<0.10$.

\section{Results}

\section{Characteristics of the cohort}

Characteristics of the cohort are reported in Table 1 (see online Table DS1 for a more detailed version); loneliness and emotional loneliness were more common in women whereas social loneliness was more prevalent in men. More women were (severely) depressed. Men had a higher prevalence of cardiovascular, cerebrovascular and pulmonary disease whereas women reported more osteoarthritis, rheumatoid arthritis, cancer and functional limitations. For peripheral artery disease and diabetes there were no differences between men and women.

\section{Loneliness, depression and gender}

When calculating interactions we found a three-way interaction (a two-way interaction that varies across levels of a third variable) between loneliness, depression and gender $(P=0.001)$, between emotional loneliness, depression and gender $(P=0.001)$ and between social loneliness, depression and gender $(P=0.01)$. Adding other variables to the model we found a three-way interaction between emotional loneliness, depression and gender $(P=0.08)$. After adding these variables no three-way interaction was found between loneliness, depression and gender $(P=0.12)$ and between social loneliness, depression and gender $(P=0.37)$.

\section{Bivariate analyses}

The overall mortality in our study was $63.5 \%(n=1828)$ at 19 years of follow-up $(70.9 \%(n=990)$ men and 56.6\% $(n=838)$ women). We found in bivariate analysis that loneliness, emotional loneliness, social loneliness and (severe) depression were all associated with excess mortality (online Table DS2). To illustrate the results we performed Kaplan-Meier analysis for the different aspects of loneliness and different depression severity levels. Online Fig. DS1 shows the Kaplan-Meier analysis for severe depression in men and women. Data for the different aspects of loneliness are not shown but showed similar results except for the gender differences, which were less prominent than in severe depression.

\section{Multivariate analyses}

We performed Cox regression analyses for the different aspects of loneliness and the different depression severity levels. All-cause mortality hazard ratios for the different aspects of loneliness and the different depression severity levels are shown in Tables 2 and 3 , respectively. We found in the final multivariate model that the different aspects of loneliness and different depression severity levels were no longer associated with excess mortality. However, in (severe) depression in men significance was only lost in the last step of analysis (shown in Table 3).

Subsequently we performed Cox proportional hazard analysis for depression and severe depression in the lonely, emotionally lonely and socially lonely subgroups. For depression we found no excess mortality in the subgroups with loneliness, emotional loneliness and social loneliness (data not shown). For severe depression we found excess mortality in men (but not in women) 


\begin{tabular}{|c|c|c|}
\hline & \multicolumn{2}{|c|}{ Mortality hazard ratios $(95 \% \mathrm{Cl})$} \\
\hline & Men & Women \\
\hline \multicolumn{3}{|l|}{ Loneliness } \\
\hline Unadjusted ratio & $1.50(1.31-1.72)$ & $1.49(1.29-1.71)$ \\
\hline Adjusted ratio & $1.13(0.97-1.32)$ & $0.99(0.85-1.16)$ \\
\hline \multicolumn{3}{|l|}{ Emotional loneliness } \\
\hline Unadjusted ratio & $1.53(1.32-1.76)$ & $1.61(1.40-1.85)$ \\
\hline Adjusted ratio & $1.04(0.88-1.23)$ & $1.12(0.95-1.31)$ \\
\hline \multicolumn{3}{|l|}{ Social loneliness } \\
\hline Unadjusted ratio & $1.17(1.02-1.35)$ & $1.33(1.13-1.56)$ \\
\hline Adjusted ratio & $1.05(0.90-1.22)$ & $1.06(0.90-1.26)$ \\
\hline
\end{tabular}

Table 3 Mortality hazard ratios for people with depression and severe depression with stepwise adjustment for potential confounders in men and women separately ${ }^{\mathrm{a}}$

\begin{tabular}{|c|c|c|}
\hline & \multicolumn{2}{|c|}{ Mortality hazard ratios $(95 \% \mathrm{Cl})$} \\
\hline & Men & Women \\
\hline \multicolumn{3}{|l|}{ Depression } \\
\hline Unadjusted ratio & $1.43(1.17-1.74)$ & $1.44(1.21-1.70)$ \\
\hline \multicolumn{3}{|l|}{ Adjustment for: } \\
\hline Demographics & $1.52(1.24-1.86)$ & $1.18(1.00-1.41)$ \\
\hline Network size & $1.50(1.22-1.84)$ & $1.18(0.99-1.40)$ \\
\hline Loneliness & $1.45(1.16-1.80)$ & $1.15(0.96-1.39)$ \\
\hline \multicolumn{3}{|l|}{ Cardiovascular and } \\
\hline cerebrovascular disease & $1.36(1.09-1.69)$ & $1.11(0.92-1.34)$ \\
\hline Other diseases & $1.29(1.03-1.61)$ & $1.11(0.92-1.34)$ \\
\hline No chronic diseases & $1.29(1.03-1.61)$ & $1.11(0.92-1.34)$ \\
\hline Mini-Mental State Examination & $1.25(1.00-1.56)^{b}$ & $1.08(0.89-1.30)$ \\
\hline Functional limitations & $1.19(0.95-1.49)$ & $0.98(0.81-1.19)$ \\
\hline \multicolumn{3}{|l|}{ Severe depression } \\
\hline Unadjusted ratio & $1.90(1.34-2.69)$ & $1.43(1.09-1.87)$ \\
\hline \multicolumn{3}{|l|}{ Adjustment for: } \\
\hline Demographics & $1.81(1.27-2.56)$ & $1.31(1.00-1.71)$ \\
\hline Network size & $1.77(1.25-2.52)$ & $1.30(0.99-1.70)$ \\
\hline Loneliness & $1.68(1.18-2.40)$ & $1.26(0.94-1.67)$ \\
\hline \multicolumn{3}{|l|}{ Cardiovascular and } \\
\hline cerebrovascular disease & $1.65(1.15-2.35)$ & $1.20(0.90-1.59)$ \\
\hline Other diseases & $1.55(1.07-2.22)$ & $1.18(0.89-1.57)$ \\
\hline No chronic diseases & $1.55(1.08-2.22)$ & $1.18(0.89-1.58)$ \\
\hline Mini-Mental State Examination & $1.53(1.06-2.20)^{\mathrm{b}}$ & $1.13(0.85-1.50)$ \\
\hline Functional limitations & $1.40(0.97-2.02)$ & $1.04(0.78-1.39)$ \\
\hline \multicolumn{3}{|c|}{$\begin{array}{l}\text { a. Hazard ratios of } 19 \text { years mortality in participants with depression and severe } \\
\text { depression; adjustment for demographics, network size, loneliness, cardiovascular } \\
\text { and cerebrovascular disease, other diseases, number of chronic diseases, cognitive } \\
\text { functioning and functional limitations. Significant hazard ratios are in bold }(P<0.05) \text {. } \\
\text { b. For men significance was only lost in the last step of analysis. }\end{array}$} \\
\hline
\end{tabular}

with both emotional and social loneliness. We found higher hazard ratios in participants who were severely depressed with social loneliness than with emotional loneliness. Results for severe depression are shown in Table 4.

To illustrate the findings in Table 4 we performed KaplanMeier analysis in subgroups of loneliness. These showed that severe depression was associated with increased mortality in men who were lonely, emotionally lonely and socially lonely with the highest excess mortality in those men who were socially lonely. In the other subgroups no increased mortality was found (see online Table DS3). Figure $1(\mathrm{a}-\mathrm{c})$ shows mortality in men with severe depression and who were lonely (a), emotionally lonely (b) and socially lonely (c), and Fig. 1(d) shows mortality for women with severe depression and who were lonely.

In conclusion, we found that the different aspects of loneliness and different depression severity levels were associated with increased mortality in bivariate analyses in both genders but not in multivariate analyses at 19 years follow-up. In the lonely subgroups we found that severe depression was associated with an increased chance of early death in men in both bivariate and multivariate analyses with the highest mortality in the socially lonely subgroup.

\section{Discussion}

\section{Main findings}

The present study shows first that emotional and social loneliness and depression are associated with excess mortality in bivariate analysis but not in multivariate analysis after 19 years of follow-up. Second, we provided evidence that severe depression is an important risk factor for death in men who are emotionally and socially lonely, with the highest risk in those men with severe depression and who are socially lonely, when also taking into account the effects of an extensive range of confounders such as cardio- and cerebrovascular diseases, cognitive functioning and functional decline, and that this association remains after 19 years of follow-up. Both loneliness and depression have been shown to be identifiable and measureable risk factors for an early death while making no distinction between emotional and social loneliness. ${ }^{8-10,12}$ Our study shows that the combination of either emotional or social loneliness with severe depression indicates a lethal combination in men in the long term. The fact that we found that depression interacts with both emotional and social loneliness is important as both conditions are open to interventions such as interpersonal and social network interventions with the emotional aspects of loneliness requiring a more interpersonal approach and social loneliness requiring an approach aimed at social networks. ${ }^{32}$

To the best of our knowledge this is the largest study of a representative general population sample of older people that examines the interplay between both emotional and social loneliness and depression and their association with excess mortality with such an extended follow-up time. Only one other study has examined the interplay between loneliness and depression with mortality as the outcome. That study showed that 85-year-old people with both depression and feelings of loneliness have a higher 5-year mortality risk whereas neither depression nor perceived loneliness as a single condition had a significant effect on mortality. ${ }^{33}$ Our findings show that these negative consequences also affect the younger old, are true for both emotional and social loneliness and are still apparent 19 years later.

\section{Explanations}

The impact of the different aspects of loneliness in combination with depression on longevity may be explained by a number of mechanisms such as suicidality and cardiovascular disease, which have been connected with both loneliness ${ }^{2,3}$ and depression. ${ }^{12,19}$ The specific mechanisms underlying the interplay between depression and loneliness and an early death are not clear, but some studies have suggested potential pathways. Stek et al ${ }^{33}$ suggest that the risk attributable to depression in the presence of perceived loneliness may result from motivational depletion and 'giving up', resulting in serious consequences such as social isolation, decreased mobility, poor diet and reduced adherence with prescribed drug regimens. Other studies specifically looked 


\begin{tabular}{|c|c|c|}
\hline \multirow[b]{2}{*}{ Subgroup } & \multicolumn{2}{|c|}{ Mortality hazard ratios $(95 \% \mathrm{Cl}), P$} \\
\hline & Men & Women \\
\hline \multicolumn{3}{|l|}{ Severe depression and loneliness } \\
\hline Unadjusted ratio & $1.60(1.10-2.34) 0.01$ & $1.08(0.78-1.48) 0.66$ \\
\hline \multicolumn{3}{|l|}{ Adjustment for: } \\
\hline Demographics & $1.65(1.13-2.41) 0.009$ & $1.21(087-1.67) 0.26$ \\
\hline Network size & $1.63(1.11-2.38) 0.01$ & $1.21(0.87-1.67) 0.26$ \\
\hline Cardiovascular and cerebrovascular disease & $1.65(1.11-2.42) 0.001$ & $1.19(0.86-1.68) 0.29$ \\
\hline Other diseases & $1.63(1.10-2.43) 0.02$ & $1.19(0.85-1.65) 0.31$ \\
\hline No chronic diseases & $1.63(1.09-2.42) 0.02$ & $1.18(0.85-1.64) 0.32$ \\
\hline Mini-Mental State Examination & $1.60(1.08-2.39) 0.02$ & $1.16(0.83-1.61) 0.39$ \\
\hline Functional limitations & $1.49(1.00-2.22) 0.05$ & $1.05(0.76-1.48) 0.75$ \\
\hline \multicolumn{3}{|l|}{ Severe depression without loneliness } \\
\hline Unadjusted ratio & $1.09(0.35-3.38) 0.89$ & $1.66(0.98-2.83) 0.06$ \\
\hline Adjusted ratio & $0.96(0.30-3.11) 0.96$ & $0.98(0.56-1.71) 0.95$ \\
\hline \multicolumn{3}{|l|}{ Severe depression and emotional loneliness } \\
\hline Unadjusted ratio & $1.58(1.08-2.32) 0.02$ & $1.14(0.83-1.55) 0.43$ \\
\hline \multicolumn{3}{|l|}{ Adjustment for: } \\
\hline Demographics & $1.78(1.22-2.62) 0.003$ & $1.16(085-1.60) 0.34$ \\
\hline Network size & $1.76(1.20-2.58) 0.004$ & $1.16(0.85-1.59) 0.35$ \\
\hline Cardiovascular and cerebrovascular disease & $1.80(1.22-2.67) 0.003$ & $1.15(0.83-1.58) 0.40$ \\
\hline Other diseases & $1.68(1.12-2.53) 0.001$ & $1.14(0.83-1.58) 0.41$ \\
\hline No chronic diseases & $1.68(1.11-2.53) 0.01$ & $1.14(0.83-1.58) 0.41$ \\
\hline Mini-Mental State Examination & $1.63(1.08-2.47) 0.02$ & $1.11(0.80-1.54) 0.52$ \\
\hline Functional limitations & $1.61(1.06-2.43) 0.02$ & $1.00(0.72-1.40) 0.97$ \\
\hline \multicolumn{3}{|l|}{ Severe depression without emotional loneliness } \\
\hline Unadjusted ratio & $1.00(0.32-3.10) 1.00$ & $1.22(0.67-2.22) 0.52$ \\
\hline Adjusted ratio & $0.76(0.24-2.44) 0.65$ & $1.21(0.66-2.21) 0.55$ \\
\hline \multicolumn{3}{|l|}{ Severe depression and social loneliness } \\
\hline Unadjusted ratio & $2.36(1.48-3.76)<0.001$ & $1.06(0.69-1.61) 0.80$ \\
\hline \multicolumn{3}{|l|}{ Adjustment for: } \\
\hline Demographics & $2.01(1.25-3.25) 0.004$ & $1.12(0.73-1.70) 0.61$ \\
\hline Network size & $1.98(1.22-3.20) 0.005$ & $1.14(0.75-1.74) 0.53$ \\
\hline Cardiovascular and cerebrovascular disease & $2.11(1.29-3.45) 0.003$ & $1.11(0.72-1.70) 0.63$ \\
\hline Other diseases & $2.47(1.48-4.14) 0.001$ & $1.11(0.72-1.71) 0.63$ \\
\hline No chronic diseases & $2.43(1.45-4.07) 0.001$ & $1.08(0.70-1.67) 0.72$ \\
\hline Mini-Mental State Examination & $2.38(1.42-4.00) 0.001$ & $1.08(0.69-1.64) 0.78$ \\
\hline Functional limitations & $2.13(1.27-3.59) 0.004$ & $0.98(0.63-1.52) 0.92$ \\
\hline \multicolumn{3}{|l|}{ Severe depression without social loneliness } \\
\hline Unadjusted ratio & $1.43(0.85-2.44) 0.18$ & $1.51(1.13-2.30) 0.009$ \\
\hline Adjusted ratio & $1.15(0.67-1.99) 0.62$ & $1.05(0.72-1.51) 0.81$ \\
\hline
\end{tabular}

at reciprocal relationships between loneliness and depression and suggest that, although loneliness is not a necessary part of depression, it is a potential risk factor for its onset. Also, reciprocal influences over time between loneliness and depressive symptomatology have been shown indicating that loneliness and depression could possibly be both a cause and an outcome. ${ }^{1}$ Social isolation could also influence the experience of depressive symptoms, and functional impairment and lower activity and participation could be both an outcome and cause of depression. ${ }^{1,34,35}$ Cacioppo et $a l^{36}$ found loneliness to be a more powerful cause of depressive symptoms in men than in women in a longitudinal study among middle-aged and older adults. Loneliness predicted changes in depressive symptoms over time and depressive symptoms predicted changes in loneliness over time, indicating that the influences between loneliness and depressive symptoms are reciprocal with a synergistic effect between these conditions. The consequences are illustrated in recent research showing that suicide attempts in older people with depression were associated with poor social problem-solving, constricted social networks and disruptive interpersonal relationships. ${ }^{37}$
We hypothesise that the combination of the different aspects of loneliness and depression may be more toxic than the sum of the two risks because the effects of loneliness and depression may add up through reciprocal influences over time. Also from an epidemiological perspective we know that an unfavourable depression course has been shown to decrease network size and increase loneliness and conversely that loneliness predicts long-term trajectories in depressive symptoms. ${ }^{38}$ This indicates once more an amplification of the co-occurrence of loneliness and depression.

We found a consistent difference between men and women in the association between the combined conditions of severe depression and (emotional and social) loneliness and excess mortality. Studies indicate significant gender differences in genetics and the immune system that play a role in a different response to stress in men compared with women. ${ }^{39}$

The exact mechanisms explaining the association between (emotional and social) loneliness, depression and mortality needs to be further explored. The combination of these conditions identifies a subgroup who are at increased risk that may need more aggressive treatment and interventions. 


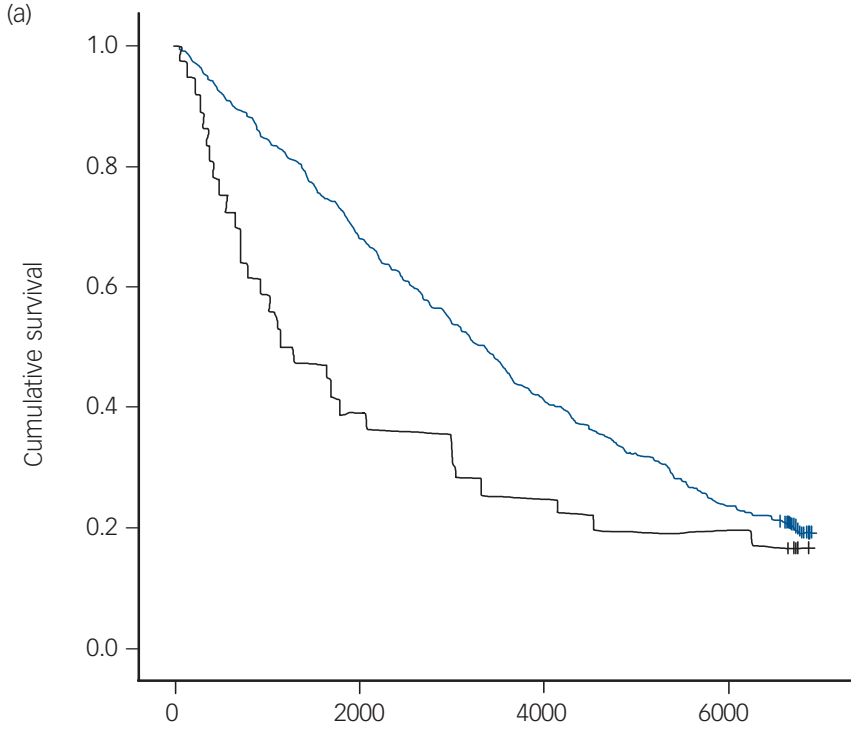

Follow-up from baseline to 1 September 2011, days

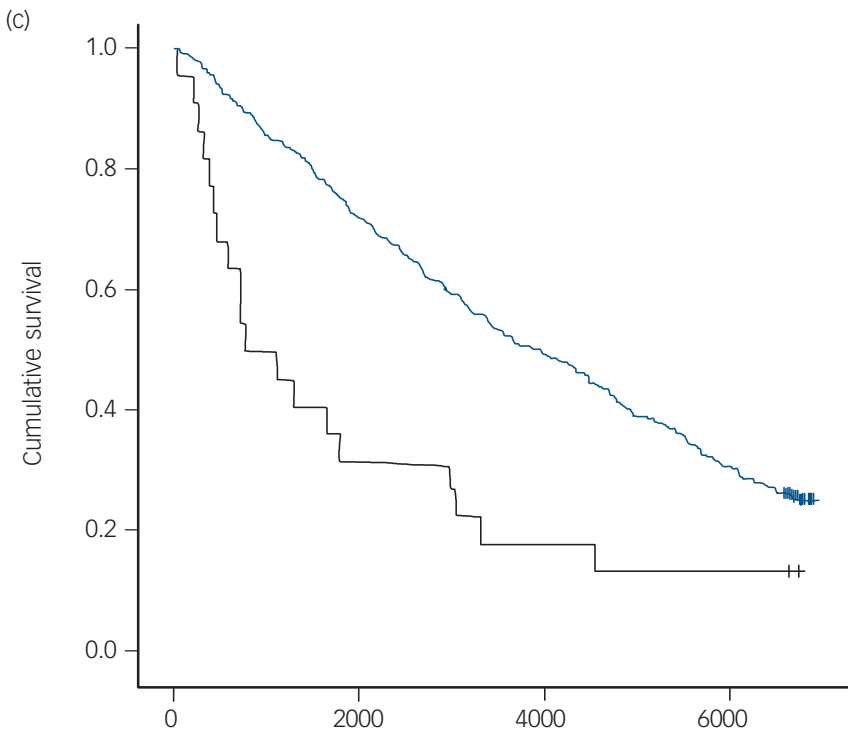

Follow-up from baseline to 1 September 2011, days

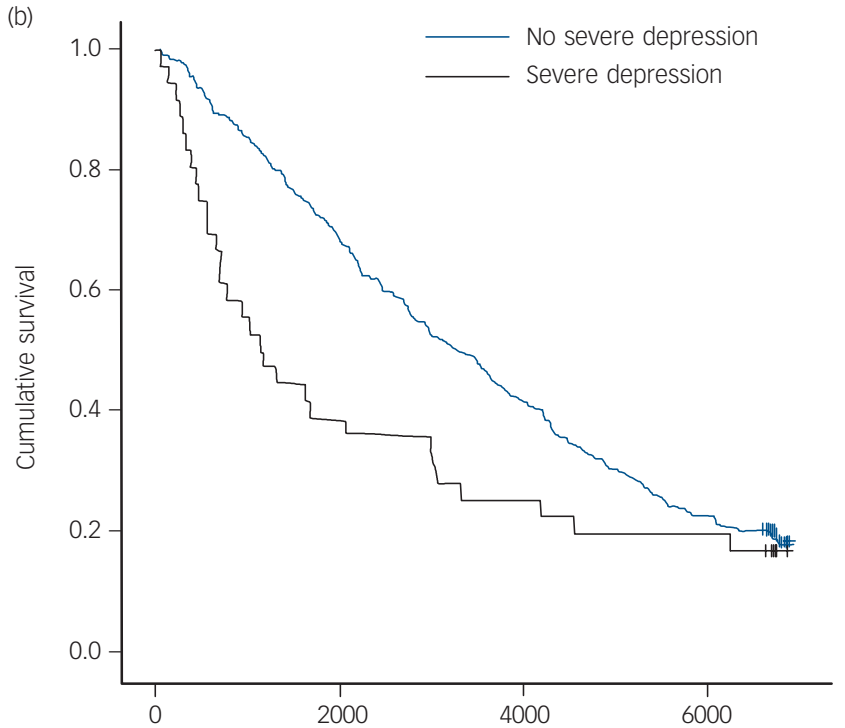

Follow-up from baseline to 1 September 2011, days

(d)

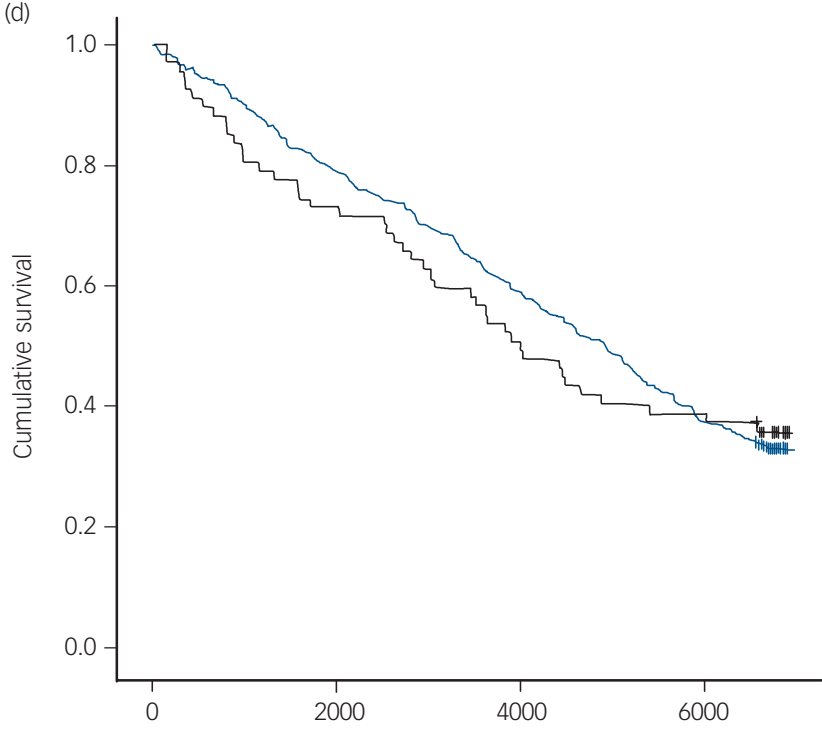

Follow-up from baseline to 1 September 2011, days

Fig. 1 Kaplan-Meier survival plots (proportion of participants who survived); cumulative survival.

(a) Men with severe depression who are lonely. Severe depression $n=36$, no severe depression $n=361$. Log rank test: $\chi^{2}=6.16$ (d.f. $=1, P=0.02$ ). (b) Men with severe depression who are emotionally lonely. Severe depression $n=36$, no severe depression $n=281$. Log rank test: $\chi^{2}=5.68$ (d.f. $=1, P=0.02$ ). (c) Men with severe depression who are socially Ionely. Severe depression $n=22$, no severe depression $n=331$. Log rank test: $\chi^{2}=13.68$ (d.f. $=1, P=0.02$ ), (d) Women with severe depression who are lonely. Severe depression $n=67$, no severe depression $n=409$. Log rank test $\chi^{2}=0.19$ (d.f. $=1, P=0.66$ ). 2000 days, 5.5 years; 4000 days, 11 years; 6000 days, 16.5 years. Vertical lines on graphs indicate censored data.

\section{Strengths}

Our study is the first study to investigate the joint effect of depression and the different aspects of loneliness on all-cause mortality in men and women. The large sample size, long follow-up, high response rate and $99.5 \%$ ascertainment of deaths are strong aspects of our study. In addition we had almost complete information on medical conditions, marital status, network size, education, cognitive functioning and functional limitations. Furthermore, both depression and loneliness were assessed with reliable and valid instruments, which is an advantage over earlier studies.

\section{Limitations}

Some limitations of the study should be considered. The information regarding medical diagnoses was based on self-report. This may lead to misclassification of the exposure variables.
However, patients' self-report on a variety of medical disorders has been shown to be reliable. ${ }^{29}$ In addition, although we adjusted for a comprehensive set of variables our study does not prove that emotional and social loneliness and depression or the combined condition are the cause of death. It is possible that the outcomes may still be explained by another unmeasured confounder. Another limitation is that we used a single observation for depression and different aspects of loneliness in our study but both the presence of depression and different aspects of loneliness could have changed during the follow-up period. Another aspect potentially affecting our findings is the fact that some symptoms of loneliness and depression overlap, which could result in overdiagnosis of loneliness, depression or both. On the other hand both conditions were measured extensively with well-established and reliable instruments that do not rely on the overlapping symptoms. Furthermore, loneliness and depression were found to have independent effects on the outcome. Although we found 
no excess mortality for depression and loneliness in multivariate analysis after 19 years of follow-up it is possible that excess mortality could be found after a shorter period of follow-up as has been shown in our earlier studies ${ }^{8,12}$ as differences in exposure variables may occur during the total follow-up period; emotional and social loneliness as well as depression may be associated with excess mortality in the short term and their combination with excess mortality in the long term. In our study children were excluded from the personal network. However, children can be an important part of the life of individuals and affect a person's perception of loneliness. Finally, another limitation is that in our study we had no information on the treatment of emotional and social loneliness and depression so the impact of treatment over time for these conditions could not be studied.

\section{Implications}

After concluding that men are more vulnerable in both their negative experience of intimacy and quality of relations with respect to the development of depression, we hypothesise that prognosis in older adults, especially men, may be improved by focusing on treatment approaches aimed at both the emotional (intimacy in relationships) and social (the number of relationships, network) aspects of loneliness and their relation with depression. Proposed interventions that showed promise in reducing loneliness in a meta-analysis ${ }^{40}$ include social skills training (aimed for example at improving conversation skills and non-verbal communication), enhancing social support (for example interventions after bereavement and relocation), increasing opportunities for social interactions, cognitivebehavioural training (focusing on maladaptive social cognition) and befriending (to develop a relationship between individuals distinct from professional-client relationships). The fact that we found that severe depression was associated with excess mortality in men meeting criteria for both emotional and social loneliness justifies interventions aimed at both types of loneliness thereby reducing both aspects of loneliness and depression by increasing emotional support and increasing the size of the network group.

Although assessment of loneliness is not usual in clinical practice, loneliness is, especially in older people, an important contributor to adverse health outcomes similar to known medical risk factors, such as cardiovascular and cerebrovascular diseases. ${ }^{10}$ It is suggested that for older men, loneliness may be just as stressful as their medical disorders. ${ }^{10}$ Loneliness can be addressed through a variety of interventions and may be treatable through these interventions just like somatic and psychiatric conditions in old age. We think for this reason that asking about the different aspects of loneliness in a structured way, especially in older men with depression, is an important task for healthcare workers in order to identify individuals at an increased risk of adverse outcomes as a consequence of loneliness.

By identifying these aspects of loneliness we will be better able to suggest interventions for loneliness. Future research needs to study potential interventions to ascertain which ones are most effective. In the meantime, healthcare professionals are advised to assess loneliness, to identify lonely older adults and to apply existing interventions. Also, older people with depression who are lonely should be referred for psychiatric, psychological and social interventions to reduce the impact of loneliness and its possible potentiating effect on depression. Recognition and treatment of depression in older adults with emotional and social loneliness may further improve social networks and interpersonal relationships, especially in men. We suggest that an integrated approach aimed at both depression and the different aspects of loneliness may improve prognosis as loneliness and depression were shown to act in a synergistic way.

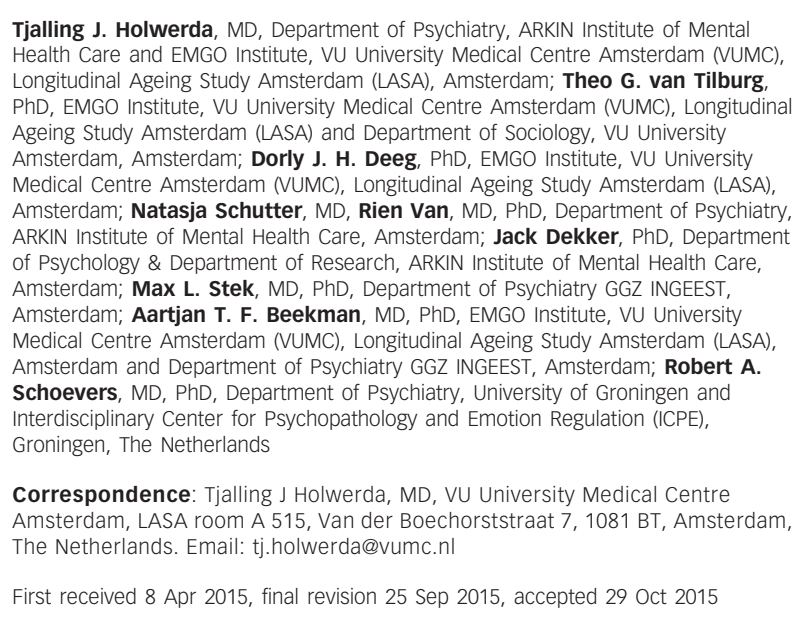

\section{Funding}

The Longitudinal Aging Study Amsterdam (LASA) was funded by the Dutch Ministry of Welfare, Health and Culture (currently Ministry of Health, Welfare and Sports).

\section{References}

1 Luanaigh CO, Lawlor BA. Loneliness and the health of older people. Int J Geriatr Psychiatry 2008; 23: 1213-21.

2 Ayalon LA, Shiovitz-Ezra S. The relationship between loneliness and passive death wishes in the second half of life. Int Psychogeriatr 2011; 23: 1677-85.

3 Thurston RC, Kubzansky LD. Women, loneliness and incident coronary heart disease. Psychosom Med. 2009; 71: 836-42.

4 Hawkley LC, Thisted RA, Masi CM, Cacioppo JT. Loneliness predicts increased blood pressure: five-year cross lagged analyses in middle-aged and older adults. Psychol Aging 2010; 25: 132-41.

5 Whisman MA. Loneliness and the metabolic syndrome in a population-based sample of middle aged and older adults. Health Psychol 2010; 29: 550-4.

6 Holwerda TJ, Deeg DJ, Beekman AT, van Tilburg TG, Stek ML, Jonker C, Schoevers RA. Feelings of loneliness but not social isolation predict incident dementia in older persons. J Neurol Neurosurg Psychiatry 2014; 85: 135-42.

7 Wilson RS, Krueger KR, Arnold SE, Schneider JA, Kelly JF, Barnes LL, Tang Y, Bennet DA. Loneliness and risk of Alzheimer Disease. Arch Gen Psychiatry 2007; 64: 234-40.

8 Holwerda TJ, Beekman AT, Deeg DJ, Stek ML, van Tilburg TG, Visser PJ, et al. Increased risk of mortality associated with social isolation in older men: only when feeling lonely? Results from the Amsterdam Study of the Elderly (AMSTEL). Psychol Med 2012; 42: 843-53.

9 Luo Y, Hawkley LC, Waite LJ, Cacioppo JT. Loneliness, health and mortality in old age: a national longitudinal study. Soc Sci Med 2012; 74: 907-14.

10 Perissinotto CM, Cenzer IS, Covinsky KE. Loneliness in older persons. A predictor of functional decline and death. Arch Intern Med 2012; 172 1078-83.

11 Hawkley LC, Cacioppo JT. Loneliness and pathways to disease. Brain Behav Immun 2003; 17 (suppl): S98-105.

12 Schoevers RA, Beckman ATF, van Tilburg W, Deeg DJH, Jonker C, Geerlings MI, et al. Association of depression and gender with mortality in old age. $\mathrm{Br} J$ Psychiatry 2000; 177: 336-42.

13 Peplau L, Perlman D. Loneliness: A Sourcebook of Current Theory, Research and Therapy. Interscience New York, 1982.

14 Weiss RS. Loneliness: The Experience of Emotional and Social Isolation. MIT Press, 1973.

15 Cacioppo S, Grippo AJ, London S, Goossens L, Cacioppo, JT. Loneliness: clinical import and interventions. Perspect Psychol Sci 2015; 10: 238-49.

16 Steptoe A, Shankar A, Demakakos P, Wardle J. Social isolation, Ioneliness, and all cause mortality in older men and women. Proc Natl Acad Sci USA 2013; 110: 5797-801. 
17 Holwerda TJ, Schoevers RA, Dekker J, Deeg DJ, Jonker C, Beekman AT. The relationship between generalized anxiety disorder, depression and mortality in old age. Int J Geriatr Psychiatry 2007; 22: 241-9.

18 Rapp MA, Gerstorf D, Helmchen $\mathrm{H}$, Smith J. Depression predicts mortality in the young old, but not in the oldest old: results form the Berlin Ageing Study. Am J Geriatr Psychiatry 2008; 16: 844-52.

19 Naqvi TZ, Naqvi SA, Merz C, Bairey N. Gender differences in the link between depression and cardiovascular disease. Psychosom Med 2005; 67 (suppl): s15-18.

20 Geerlings SW, Beekman AT, Deeg DJ, Twisk JW, Van Tilburg W. Duration and severity of depression predict mortality in older adults in the community. Psychol Med 2000; 32: 609-18.

21 Huisman M, Poppelaars J, Van der Horst M, Beekaman ATF, Brug J, Van Tilburg TG, et al. Cohort profile: the Longitudinal Ageing Study Amsterdam. Int J Epidemiol 2011; 40: 868-76.

22 De Jong Gierveld J, Kamphuis FH. The development of a Rasch-type Ioneliness-scale. Appl Psychol Meas 1985; 9: 289-99.

23 Van Tilburg TG, De Jong Gierveld J. Reference standards for the loneliness scale. Tijdschr Geront Geriatr 1999; 30: 158-63.

24 Radloff LS. The CES-D Scale: a self-report depression scale for research in the general population. Appl Psychol Meas 1977; 1: 385-401.

25 Beekman AT, Deeg DJ, Van Limbeek J, Braam AW, De Vries MZ, Van Tilburg W. Criterion validity of the Center for Epidemiological Studies Depression Scale (CES-D). Results from a community-based sample of older subjects in the Netherlands. Psychol Med 1997; 27: 231-5.

26 Chabrol H, Montovany A, Chouicha K, Duconge E. Study of the CES-D on a sample of 1953 adolescent students. Encephale 2002; 28: 429-32.

27 Lesman-Leegte I, Van Veldhuisen DJ, Hillege HL, Moser D, Sanderman R, Jaarsma T. Depressive symptoms and outcomes in patients with heart failure: data from the COACH study. Eur J Heart Fail 2009; 11: 1202-7.

28 van Tilburg TG. Losing and gaining in old age: changes in personal network size and social support in a four-year longitudinal study. J Gerontol Soc SCi 1998; 53B: S313-23.
29 Kriegsman DMW, Penninx BWJH, Van Eijk JTM. Self-reports and general practioner information on the presence of chronic diseases in community dwelling elderly. A study on the accuracy of patients' self reports and on determinants of inaccuracy. J Clin Epidemiol 1996; 49: 1407-17.

30 Folstein MF, Folstein SE, McHugh PR. Mini-mental state: a practical method for the clinician. J Psychiatr Res 1975; 12:189-98.

31 McWhinnie JR. Disability assessment in population surveys: results of the OECD common development effort. Rev Epidemiol Santé Publique 1981; 29: 413-9.

32 Van Tilburg TG, De Jong Gierveld J. Zicht Op Eenzaamheid. Achtergronden, Oorzaken En Aanpak. Van Gorcum Assen, 2007.

33 Stek ML, Vinkers DJ, Gussekloo J, Beekman ATF, Van der Mast RC, Westendorp RGJ. Is depression in old age fatal only when people feel lonely? Am J Psychiatry 2005; 162: 178-80.

34 Alpass FM, Neville S. Loneliness, health and depression in older males. Aging Mental Health 2003; 7: 212-6.

35 Adams KB, Sanders S, Auth EA. Loneliness and depression in independent living retirmement communities: risk and resilience factors. Aging Mental Health 2004; 8: 475-85.

36 Cacioppo JT, Hughes ME, Waite LJ, Hawkley LC, Thisted A. Loneliness as a specific risk factor for depressive symptoms: cross sectional and longitudinal analysis. Psychol Aging 2006; 21: 140-51.

37 Szanto K, Dombrovski AY, Sahakian BJ, Mulsant BH, Houck PR, Reynolds CF 3rd. Social emotion recognition, social functioning and attempted suicide in late life depression. Am J Geriatr Psychiatry 2012; 20: 257-65.

38 Houtjes W, van Meijel B, van de Ven PM, Deeg D, van Tilburg T, Beekman A. The impact of an unfavorable depression course on network size and Ioneliness in older people: a longitudinal study in the community. Int J Geriatr Psychiatry 2014; 29: 1010-7.

39 Choi BG, McLaughlin MA. Why men's hearts break: cardiovascular effects of sex steroids. Endocrinol Metab Clin North Am 2007; 36: 365-77.

40 Masi CM, Chen HY, Hawkley LC, Cacioppo JT. A meta-analysis of interventions to reduce loneliness. Pers Soc Psychol Rev 2011; 15; 219-66. 